\title{
Health status measured by Kansas City Cardiomyopathy Questionnaire-12 in primary prevention implantable cardioverter defibrillator patients with heart failure
}

\author{
Gustav Mattsson ${ }^{1 *}$ (D) Marita Wallhagen ${ }^{2}$ (i) and Peter Magnusson ${ }^{1,3}$ (B)
}

\begin{abstract}
Background: Self-reported health status as measured by the Kansas City Cardiomyopathy Questionnaire (KCCQ) in patients with primary prevention implantable cardioverter defibrillators (ICDs) has mainly been reported from randomized trials. However, these studies are often limited to short follow-up and are subject to selection bias. The aim of this study was to assess KCCQ-12 in patients with primary prevention ICD due to either ischemic or nonischemic heart failure.
\end{abstract}

Methods: This cross-sectional observational study included all patients in Region Gävleborg, Sweden, who because of primary prevention due to heart failure, had an ICD or underwent device replacement between 2007 and 2017. After validation using medical records patients were sent and returned the KCCQ-12 by regular mail.

Results: A total of 118 questionnaires were analyzed (response rate $71.1 \%$ ). The mean age was $70.9 \pm 9.8$ years, and a minority was female $(n=20,16.9 \%)$. The mean overall summary score was $71.5 \pm 22.4$, there was no significant difference between ischemic and nonischemic heart failure ( $69.5 \pm 23.1 \mathrm{vs.} 74.4 \pm 21.3 ; p=0.195)$. Atrial fibrillation at baseline was associated with lower score for the domains Symptom frequency $(70.2 \pm 23.2 \mathrm{vs} .82 .2 \pm 19.2 ; p=0.006)$ and Social limitation $(62.1 \pm 26.0$ vs. $75.6 \pm 26.6 ; p=0.006)$ as well as the overall summary score $(63.9 \pm 21.3 \mathrm{vs.} 74.8 \pm 22.2$; $p=0.004$ ).

Conclusion: In a real-world setting, primary prevention ICD patients with heart failure report an acceptable diseasespecific health status at long-term follow-up. Ischemic and nonischemic etiology showed similar health status whereas atrial fibrillation was associated with worse outcome.

Keywords: Cardiomyopathy, Heart failure, Implantable cardioverter defibrillator, Kansas City Cardiomyopathy Questionnaire, Quality of life

*Correspondence: gustav.mattsson@regiongavleborg.se

${ }^{1}$ Centre for Research and Development, Uppsala University/Region

Gävleborg, 80187 Gävle, Sweden

Full list of author information is available at the end of the article

\section{Background}

An implantable cardioverter defibrillator (ICD) increases survival in those at high risk for sudden cardiac death [1]. An ICD protects from life-threatening arrhythmias by cardioversion, antitachycardia pacing or bradycardia pacing. In combination with a left-ventricular lead it can also offer cardiac resynchronization therapy (CRT-D) for heart failure in selected cases [2]. Guidelines from 
the European Society of Cardiology (ESC) recommend a primary prophylactic ICD for patients with symptomatic heart failure with New York Heart Association functional classification (NYHA-class) II-III, left ventricular ejection fraction $\leq 35 \%$ despite at least three months of optimal medical therapy, and a life expectancy of at least 1 year with good functional status [1].

Patient reported outcome measures through questionnaires have been increasingly used in research settings [3]. In patients with heart failure, the Kansas City Cardiomyopathy Questionnaire (KCCQ) offers a diseasespecific measurement of health status and health-related quality of life [4]. The 12-item KCCQ, the KCCQ-12 preserves the psychometric properties of the original questionnaire, shows high correlation with the original scales ( $>0.93$ for all scales in all clinical settings), hightest-retest reliability ( $>0.76$ for all domains), and high responsiveness to clinical change [5]. The $\mathrm{KCCQ}$, as well as KCCQ-12 correlate with physician assessed NYHAclass as well as heart failure outcomes [4-6].

The primary aim of this study was to, with a cross-sectional observational design, assess self-reported health status as measured by the KCCQ-12 in an unselected cohort of patients with primary prevention ICD due to ischemic or nonischemic heart failure without tertiary center bias. The secondary aims were to assess whether self-reported health status as measured by the KCCQ12 were affected by etiology of heart failure (ischemic vs nonischemic), atrial fibrillation before implant, age, sex, complications requiring surgery, and appropriate therapy or inappropriate shock.

\section{Methods \\ Data collection and validity}

This cross-sectional observational study included patients from Region Gävleborg in Sweden who, because of primary prevention due to heart failure, had an ICD implanted or who underwent device replacement between 1st January 2007 and 1st of January 2017. Patients were identified from a retrospective observational study. Long-term outcome with regard to appropriate therapy, inappropriate shock, complications requiring surgery, mortality, and cause of death in this cohort has previously been reported [7]. Information about baseline characteristics as well as appropriate therapy, inappropriate shock, complications requiring surgery, and mortality at long-term follow up had been retrieved from electronic medical records (Melior ${ }^{\mathrm{TM}}$, Cerner Sverige AB, Stockholm) between March 2017 and February 2018. Eligible patients with primary prevention ICD due to heart failure who had a Swedish postal address were sent and returned a Swedish version of the KCCQ-12 by regular mail during 2019.

\section{The KCCQ-12}

In the KCCQ-12, responses are given on a Likert scale that for each individual item is scored on a scale of 0-100 with higher scores indicating better health. Items are grouped into the four domains; Physical limitation, Symptom frequency, Quality of life, and Social limitation. The score of each domain is calculated as the average of its constituent items. In case of missing values for an item, the score of that item is considered to be the same as the average of the other items in its domain. An overall summary score is calculated as the average of all four domains.

\section{Statistical analyses}

Data were described as frequencies, percentages, and means including standard deviations $( \pm)$. The $t$-test was used for comparisons of normally distributed continuous variables. The Mann-Whitney U-test was used for non-normally distributed continuous variables. The Shapiro-Wilk test was used to test for the normality distribution of data. The chi-squared test was used for comparisons of categorical variables. Differences in KCCQ-12 domains and summary scores between groups were tested with Mann-Whitney U-test. The associations between age and KCCQ-12 were tested using Spearman's rank-order correlation. Kruskal-Wallis non-parametric analysis of variance was used to analyze differences between the age strata; 32-59 years, 60-69 years, $70-79$ years, and $\geq 80$ years. The magnitude of difference in KCCQ-12 domains or summary score between groups was reported as the difference between the means. Two-sided $p$ values $\leq 0.05$ were considered statistically significant. The software programs Excel 2010 (Microsoft Corporation, Redmond, WA), SPSS version 22 (IBM, Armonk, NY) were used for analyses.

\section{Results \\ Response rate and dropout analysis}

Out of 236 patients identified with primary prevention ICD implantation due to heart failure, 169 were still alive at the time of health status assessment. Out of these 169 patients, 166 had a Swedish postal address and were sent the KCCQ-12 by regular mail including a pre-paid return envelope. The questionnaire was returned by 123 patients, in 118 of these all domains could be calculated. Figure 1 depicts the process for deriving the final sample for analysis. The response rate was $71.1 \%$. The age of those that returned complete questionnaires was similar to those that did not $(70.9 \pm 9.8$ vs. $67.0 \pm 12.45$ years; $p=0.062$ ). The proportion of females was lower among 


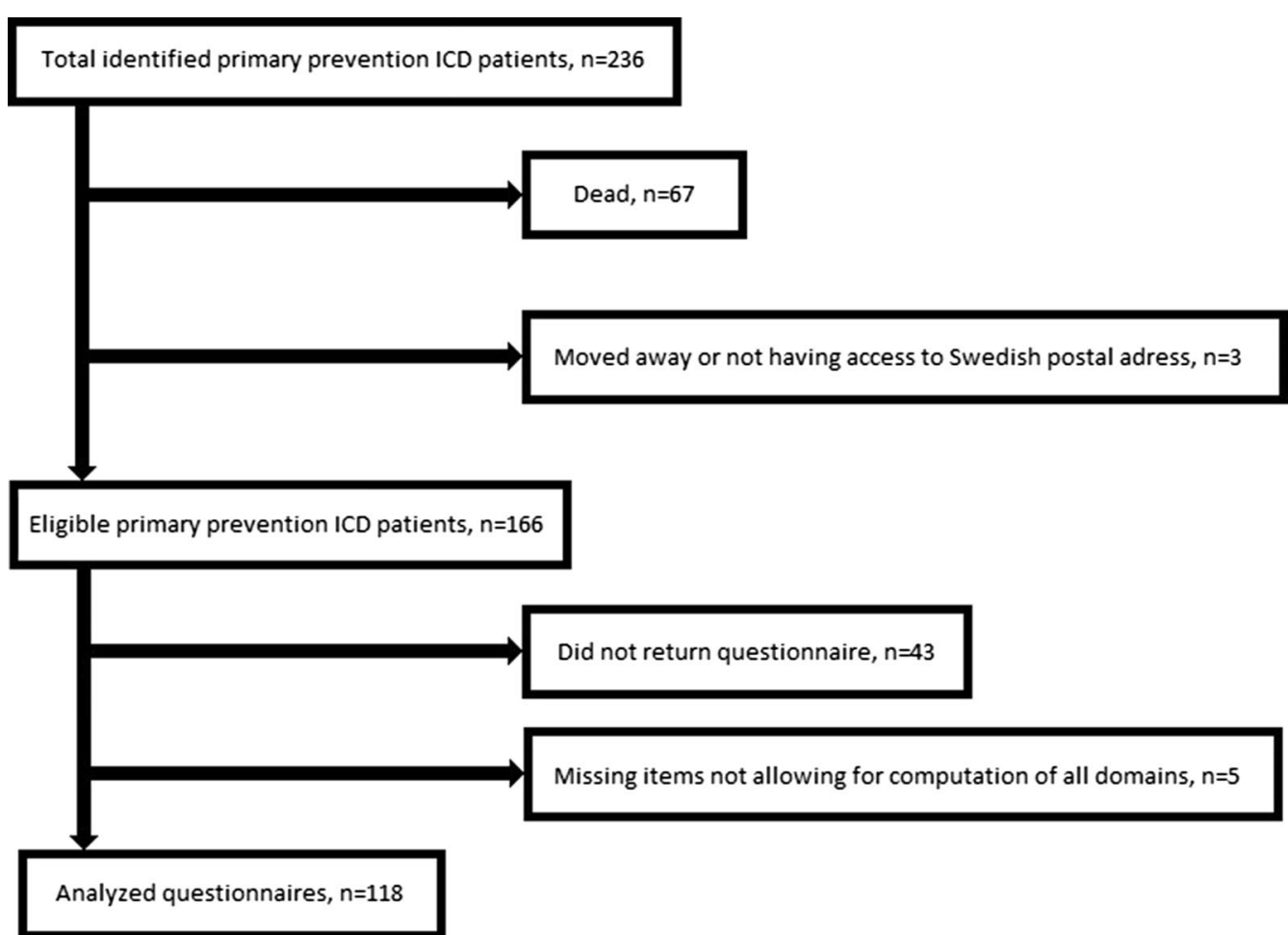

Fig. 1 Flow-chart depicting the process for deriving the final sample for analysis

those who returned a complete questionnaire than for those that did not $(16.9 \%$ vs. $22.9 \% ; p<0.001)$.

\section{Cohort characteristics at ICD implantation}

The baseline characteristics of the sample $(n=118)$ at ICD implantation are summarized in Table 1 . The mean age at ICD implantation was $65.0 \pm 10.4$ years. A minority was female $(n=20,16.9 \%)$. The mean age at the time of the survey was similar between females and males (71.2 \pm 7.8 vs. $70.9 \pm 10.1$ years; $p=0.904)$. Almost half of the patients had a CRT-D at first implantation $(n=57$, 48.3\%). A majority of patients had ischemic etiology $(\mathrm{n}=69,58.5 \%)$ and patients with ischemic etiology was older than patients with nonischemic etiology $(73.3 \pm 8.1$ vs. $67.6 \pm 10.9 ; p=0.005)$. Patients who had atrial fibrillation at baseline were older at the time of the survey than those that did not $(74.4 \pm 8.1$ vs. $69.5 \pm 10.1 ; p=0.013)$.

\section{Cohort characteristics at follow-up}

In the final sample, of the 118 patients, the mean time between ICD implantation and follow-up with KCCQ12 was $5.9 \pm 2.3$ years. The mean time from ICD implantation to evaluation of baseline characteristics and outcomes such appropriate therapy, inappropriate shock, and complications requiring surgery was $4.1 \pm 2.4$ years. Out of these 118 patients; 16 (13.6\%) experienced
Table 1 Characteristics at ICD implant of the 118 patients analyzed regarding KCCQ-12

\begin{tabular}{ll}
\hline & $\mathbf{n}(\%)$ \\
\hline Patients & 118 \\
Mean age at implant (years) & $65.0 \pm 10.4$ \\
Mean age at KCCQ (years) & $70.9 \pm 9.8$ \\
Females & $20(16.9)$ \\
Ischemic etiology & $69(58.5)$ \\
Device type & \\
ICD-VR & $16(13.6)$ \\
ICD-DR & $45(38.1)$ \\
CRT-D & $57(48.3)$ \\
Hypertension & $54(45.8)$ \\
Diabetes mellitus & $27(22.9)$ \\
Renal failure* & $16(13.6)$ \\
Atrial fibrillation & $35(29.7)$ \\
Beta-blockers & $106(89.8)$ \\
ACE-i/ARB & $111(94.1)$ \\
MRA & $75(63.6)$ \\
\hline
\end{tabular}

Data presented as frequencies (percentage in parenthesis)

$A C E-i$ angiotensin converting enzyme inhibitor, $A R B$ angiotensin receptor blockers, $C R T-D$ cardiac resynchronization therapy defibrillator, ICD-DR dual lead implantable cardioverter defibrillator, ICD-VR single lead implantable cardioverter defibrillator, KCCQ-12 12-item Kansas City Cardiomyopathy Questionnaire, MRA mineralcorticoid receptor antagonists

*Defined as S-Creatinine $\geq 130 \mu \mathrm{mol} / \mathrm{L}$ 
appropriate ICD therapy (defined as antitachycardia pacing or cardioversion of ventricular tachyarrhythmia), 5 (4.2\%) experienced inappropriate shock (defined as cardioversion in the absence of ventricular tachyarrhythmia), and 16 (13.6\%) experienced at least one complication requiring surgical intervention. The mean age was similar for those that experienced appropriate therapy vs those that did not ( $72.9 \pm 8.1$ vs. $70.6 \pm 10.0$ years; $p=0.376)$, for those that experienced inappropriate shock vs those that did not (73.4 \pm 8.4 vs. $70.8 \pm 9.8 ; p=0.563)$, and for those that experienced complications requiring surgery vs those that did not $(74.7 \pm 8.2$ vs. $70.3 \pm 9.9 ; p=0.099)$.

\section{Health status measured by KCCQ-12}

The results for the four domains and the summary score of the KCCQ-12, for all patients as well as for patients with heart failure of ischemic and nonischemic etiology is given in Table 2. The mean of the overall summary score for all patients was $71.5 \pm 22.4$. There was no statistically significant difference between ischemic and nonischemic heart failure $(69.5 \pm 23.1$ vs. $74.4 \pm 21.3 ; p=0.195)$ with regards to the overall summary score, nor was there any statistically significant difference for any domain. In Table 2 the results of the four domains and the summary score are presented for patients with ICD-VR or ICD-DR as compared to patients with CRT-D. The overall summary score was lower in patients with CRT-D compared to ICD-VR/DR $(67.7 \pm 22.4$ vs. $75.1 \pm 22.0 ; p=0.041)$. Patients with CRT-D reported lower scores in the domain Symptom frequency $(74.8 \pm 21.2$ vs. $82.3 \pm 20.5$; $p=0.040)$.

\section{Age}

There was a negative correlation between age at the time of the survey and overall summary score (Spearman's correlation coefficient, $\left.\mathrm{r}_{\mathrm{S}}=-0.186 ; p=0.043\right)$, Physical limitation $\left(\mathrm{r}_{\mathrm{S}}=-0.230 ; p=0.012\right)$, and Symptom frequency $\left(\mathrm{r}_{\mathrm{S}}=-0.252 ; p=0.006\right)$. But no correlation between age and the Quality of life $(p=0.418)$ or Social limitation $(p=0.192)$ domains.

The KCCQ-12 scores for the age strata; $32-59$ years, 60-69 years, $70-79$ years, and $\geq 80$ years as well as the result of the Kruskal-Wallis tests are shown in Table 3. There was a significant effect of age strata upon Symptom frequency $(p=0.046)$. However, for the youngest age strata, scores for this domain was slightly lower $(80.1 \pm 22.1)$ than for the second youngest age strata $(84.7 \pm 19.7)$, while after that scores decreased with increasing age with age strata $70-79$ years scoring $75.7 \pm 19.6$ and age strata $\geq 80$ years $72.5 \pm 23.8$. There was no statistically significant difference between the age strata for Physical limitation, Quality of life, or Social limitation.

\section{Sex}

There was no significant difference between females and males for the overall summary score $(71.1 \pm 24.2$ vs. $71.6 \pm 22.1 ; p=0.919)$ or for any of the separate domains; Physical limitation $(p=0.679)$, Symptom frequency $(p=0.455)$, Quality of life $(0.858$, or Social limitation $(p=0.992)$.

Table 2 Mean score of the KCCQ-12 in 118 patients with primary prevention ICD due to heart failure

\begin{tabular}{|c|c|c|c|c|}
\hline KCCQ-12 & $\begin{array}{l}\text { All } \\
n=118 \\
\text { Mean score }\end{array}$ & $\begin{array}{l}\text { Ischemic HF } \\
n=69 \\
\text { Mean score }\end{array}$ & $\begin{array}{l}\text { Nonischemic HF } \\
n=49 \\
\text { Mean score }\end{array}$ & $\begin{array}{l}\text { Ischemic versus } \\
\text { nonischemic HF } \\
p \text { value }\end{array}$ \\
\hline Physical limitation & $68.0 \pm 26.4$ & $64.6 \pm 26.2$ & $72.8 \pm 26.1$ & 0.062 \\
\hline Symptom frequency & $78.6 \pm 21.1$ & $77.2 \pm 21.5$ & $80.6 \pm 20.5$ & 0.305 \\
\hline Quality of life & $69.7 \pm 25.7$ & $68.8 \pm 25.7$ & $70.9 \pm 25.8$ & 0.582 \\
\hline Social limitation & $71.6 \pm 27.0$ & $68.8 \pm 27.1$ & $75.6 \pm 26.7$ & 0.144 \\
\hline Overall summary score & $71.5 \pm 22.4$ & $69.5 \pm 23.1$ & $74.4 \pm 21.3$ & 0.195 \\
\hline KCCQ-12 & $\begin{array}{l}\text { All } \\
n=118 \\
\text { Mean score }\end{array}$ & $\begin{array}{l}\text { ICD-VR/DR } \\
\mathrm{n}=61 \\
\text { Mean score }\end{array}$ & $\begin{array}{l}\text { CRT-D } \\
n=57 \\
\text { Mean score }\end{array}$ & $\begin{array}{l}\text { ICD-VR/DR } \\
\text { versus CRT-D } \\
p \text { value }\end{array}$ \\
\hline Physical limitation & $68.0 \pm 26.4$ & $72.0 \pm 23.3$ & $63.7 \pm 28.9$ & 0.158 \\
\hline Symptom frequency & $78.6 \pm 21.1$ & $82.3 \pm 20.5$ & $74.8 \pm 21.2$ & 0.040 \\
\hline Quality of life & $69.7 \pm 25.7$ & $73.2 \pm 25.2$ & $66.0 \pm 25.9$ & 0.093 \\
\hline Social limitation & $71.6 \pm 27.0$ & $75.0 \pm 27.5$ & $68.0 \pm 26.3$ & 0.096 \\
\hline Overall summary score & $71.5 \pm 22.4$ & $75.1 \pm 22.0$ & $67.7 \pm 22.4$ & 0.041 \\
\hline
\end{tabular}

Statistically significant $p$-values are given in bold

Comparisons are made between ischemic versus nonischemic HF and VR/DR versus CRTD

$C R T-D$ cardiac resynchronization therapy defibrillator, HF heart failure, ICD implantable cardioverter defibrillator, ICD-VR/DR single or dual lead implantable cardioverter defibrillator, KCCQ-12 12-item Kansas City Cardiomyopathy Questionnaire, SD standard deviation 
Table $3 \mathrm{KCCQ}-12$ score in 118 patients with primary prevention ICD due to heart failure stratified by age

\begin{tabular}{llllll}
\hline KCCQ-12 & $\begin{array}{l}\text { 32-59 years }(\mathbf{n}=\mathbf{1 0}) \\
\text { Mean score }\end{array}$ & $\begin{array}{l}\mathbf{6 0 - 6 9} \text { years }(\mathbf{n = 4 2 )} \\
\text { Mean score }\end{array}$ & $\begin{array}{l}\mathbf{7 0 - 7 9} \text { years }(\mathbf{n}=\mathbf{4 2}) \\
\text { Mean score }\end{array}$ & $\begin{array}{l}\mathbf{8 0} \text { years }(\mathbf{n}=\mathbf{2 4}) \\
\text { Mean score }\end{array}$ & $\begin{array}{l}\text { Kruskal-Wallis test } \\
\boldsymbol{p} \text { value }\end{array}$ \\
\hline Physical limitation & $75.8 \pm 8.1$ & $71.7 \pm 24.9$ & $67.5 \pm 26.7$ & $59.0 \pm 27.5$ & 0.164 \\
Symptom frequency & $80.1 \pm 22.1$ & $84.7 \pm 19.7$ & $75.7 \pm 19.6$ & $72.5 \pm 23.8$ & $\mathbf{0 . 0 4 6}$ \\
Quality of life & $66.3 \pm 28.3$ & $72.9 \pm 27.2$ & $67.3 \pm 24.4$ & $69.8 \pm 25.0$ & 0.520 \\
Social limitation & $82.5 \pm 22.7$ & $72.0 \pm 28.1$ & $70.1 \pm 25.1$ & $69.1 \pm 30.4$ & 0.564 \\
Overall summary score & $75.6 \pm 22.6$ & $74.4 \pm 22.9$ & $69.6 \pm 21.7$ & $68.2 \pm 23.0$ & 0.402 \\
\hline
\end{tabular}

Statistically significant $p$-values is given in bold

ICD implantable cardioverter defibrillator, KCCQ-12 12-item Kansas City Cardiomyopathy Questionnaire

\section{Subgroup analysis of the KCCQ-12}

Subgroup analyzes for atrial fibrillation, appropriate therapy, inappropriate shock, and complications requiring surgery are shown in Table 4. Patients with a history of atrial fibrillation before ICD implant had at followup significantly lower score for the domains Symptom frequency $(70.2 \pm 23.2$ vs. $82.2 \pm 19.2 ; p=0.006)$ and Social limitation $(62.1 \pm 26.0$ vs. $75.6 \pm 26.6 ; p=0.006)$ as well as the overall summary score $(63.9 \pm 21.3$ vs. $74.8 \pm 22.2 ; p=0.004)$, but there was no statistically significant difference in the scores for the domain Quality of life $(p=0.119)$. However, for the domain Physical limitation there was a trend toward significance $(p=0.051)$ with atrial fibrillation patients having lower scores $(61.0 \pm 27.3$ vs. $70.9 \pm 25.6)$. Patients with and without a history of appropriate therapy or complications requiring surgery had similar scores on all KCCQ-12 domains and the overall summary score. There were few patients who had received inappropriate therapy $(n=5)$. Patients who had received inappropriate therapy had significantly lower scores for both the domain Social limitation ( $43.3 \pm 19.0$ vs. $72.5 \pm 22.1$; $p=0.015)$ and the overall summary score $(50.7 \pm 19.0$ vs. $72.5 \pm 22.1 ; p=0.031$ ), but there was no statistically significant differences in the domains Physical limitation, Symptom frequency, or Quality of life.

\section{Discussion}

In real-world primary prevention ICD cohorts, KCCQ12 scores might be lower than in cohorts reported from randomized trials. However, KCCQ-12 scores in this population considering the long-term follow-up appear to be generally acceptable, indicating selection of patients with high functional status at implantation. A change of at least 5 points in KCCQ-12 scores is usually considered to be the minimal clinically important difference $[5,8]$.

The short form of the KCCQ, the KCCQ-12 was created as a tool for easier implementation into clinical practice and preserves the psychometric properties of the original KCCQ [5]. For this reason, the KCCQ-12 was used in this study, however this makes comparisons to some studies more difficult. In the Multicenter Automatic Defibrillator Implantation Trial With Cardiac Resynchronization Therapy (MADIT) trial, health status was measured with the $\mathrm{KCCQ}$, due to reporting of all separate domains, an approximation of the KCCQ-12 overall summary score can be calculated [9]. At inclusion the mean of the overall summary score for the 675 patients with only ICD was 75.2 for all KCCQ domains and 75.1 for all KCCQ-12 domains, for the 1024 patients with CRT-D the mean of the overall summary score was 75.6 for all KCCQ domains and 75.4 for all KCCQ-12 domains [9]. This demonstrates how similar the overall

Table 4 Subgroup analyses of KCCQ-12 scores in 118 patients with primary prevention ICD due to heart failure

\begin{tabular}{|c|c|c|c|c|c|c|c|c|}
\hline \multirow[t]{2}{*}{ KCCQ-12 } & \multicolumn{2}{|l|}{$\begin{array}{l}\text { Atrial fibrillation } \\
n=35\end{array}$} & \multicolumn{2}{|c|}{$\begin{array}{l}\text { Appropriate therapy } \\
n=16\end{array}$} & \multicolumn{2}{|c|}{$\begin{array}{l}\text { Inappropriate shock } \\
n=5\end{array}$} & \multicolumn{2}{|l|}{$\begin{array}{l}\text { Complications } \\
\mathrm{n}=16\end{array}$} \\
\hline & Mean difference & $p$ value & $\begin{array}{l}\text { Mean } \\
\text { difference }\end{array}$ & $p$ value & Mean difference & $p$ value & Mean difference & $p$ value \\
\hline Physical limitation & -10.0 & 0.051 & 9.9 & 0.238 & -17.0 & 0.187 & 4.5 & 0.782 \\
\hline Symptom frequency & -12.0 & 0.006 & 2.4 & 0.946 & -18.6 & 0.098 & -0.4 & 0.864 \\
\hline Quality of life & -8.2 & 0.119 & 0.7 & 0.795 & -18.0 & 0.168 & 2.5 & 0.898 \\
\hline Social limitation & -13.5 & 0.006 & 3.9 & 0.958 & -29.6 & 0.015 & 1.5 & 0.930 \\
\hline Overall summary score & -10.9 & 0.004 & 3.6 & 0.841 & -21.8 & 0.031 & 2.0 & 0.925 \\
\hline
\end{tabular}

Statistically significant $p$-values are given in bold

Positive mean difference indicates higher values

ICD implantable cardioverter defibrillator, KCCQ-12 12-item Kansas City Cardiomyopathy Questionnaire 
summary score is for KCCQ and KCCQ-12, in a population with primary prevention ICD due to heart failure, the removal of the domains symptom stability and symptom burden does not affect the overall summary score.

The MADIT included patients with left ventricular ejection fraction $\leq 30 \%$ but only NYHA class I-II. At follow-up after three years, the mean KCCQ overall summary score for left bundle branch block patients solely with ICD was 80.0 and for patients with CRT-D was 83.4 [9]. Both higher than the mean KCCQ-12 overall summary score of 71.5 in our cohort after a mean follow-up of 5.9 years. This is partly explained by the inclusion of only NYHA class I-II in the MADIT while primary prevention ICD guidelines recommend implantation of an ICD for NYHA II-III [1]. In the MADIT patients were randomized to the intervention of CRT-D implantation and CRT-D was shown to increase KCCQ scores. In our study, patients where not randomized to CRT-D. In real world cohorts patients with more severe symptoms might be more prone to receive CRT-D. This likely explains why patients with CRT-D had lower KCCQ scores in our cohort.

In the Danish Study to Assess the Efficacy of Implantable Cardioverter-Defibrillators (DANISH) trial heart failure patients were randomized to either ICD or usual clinical care and health status was measured using the Minnesota Living with Heart Failure Questionnaire (MLHFQ) [10]. After 32 months the change in mean MLHFQ overall score was similar between the ICD and control group $(p=0.82)$. Probably differences between heart failure patients with and without primary prevention ICD are primarily not caused by ICD implantation but rather by the selection process.

\section{Age}

There were moderate correlations between age and the KCCQ-12 domains Physical limitation, Symptom frequency, and the overall summary score. This was strongest for Symptom frequency $(\mathrm{rS}=-0.252)$, that also was the domain that showed significant differences between the prespecified age strata. It is likely that Quality of life and Social limitation, domains that did not show statistically significant correlations with age, reflects more of a subjective interpretation of health-related quality of life, while domains such as Physical limitation and Symptom frequency reflect more of an objective assessment of heart failure specific health status that is more age-dependent. It is also likely that physicians offer all younger patients that fulfill guideline criteria a primary prevention ICD, while for older patients there is a selection, where those patients who have higher health-related quality of life are more likely to have an ICD implanted. A large study of outpatient heart failure patients showed a small negative effect (mean difference-5.5 per 10-year increment, adjusted for sex and ethnicity) of age upon KCCQ overall summary score for those patients who are $\geq 70$ years while this was not seen for patients $<70$ years of age [11].

\section{Sex}

The proportion of females was notably low (16.9\%), this was partly due to females in our cohort being somewhat less likely to return the questionnaire as was seen in the drop-out analysis with chi-squared test $(p<0.001)$, but also due to a low proportion of females among eligible participants for the study in the cohort of primary prevention ICD patients (22.9\%). The low proportion of females in primary prevention ICD cohorts is wellknown, in one study with pooled data from eleven European national registries the proportion of females was $18.7 \%$ [12]. In our study sex category had no effect on any of the KCCQ-12 domains or overall summary score. In heart failure, differences between the sexes in healthrelated quality of life and health status are controversial. While results of many studies have been heterogeneous, some have reported lower KCCQ scores in female than in male heart failure patients, independently from other clinical factors $[11,13]$. The reason for lower proportions of women in ICD cohorts is unclear, females with primary prevention ICD has been shown to have a lower mortality and lower risk of appropriate therapy than males [12]. Therefore, sex differences in heart failure health status or health-related quality of life, if any exist, might differ between general heart failure populations and primary prevention ICD cohorts.

\section{Appropriate therapy}

Previous prospective observational studies have identified ICD shocks as a factor decreasing health-related quality of life and physical activity as well as increasing anxiety [14]. In our study no significant association between appropriate therapy and health status measured by the KCCQ-12 was seen. In the PainFree SST clinical trial, anxiety remained increased for the 24 month follow-up after a shock, reduction in daily activity was seen but returned to normal after 3 months [14]. Our patients with a longer time with an ICD (5.9 \pm 2.3 years) might reflect a population in which health status no longer is affected by previous ICD therapy. However, the lack of a statistically significant association between appropriate therapy and health status might also be due to our lower sample size resulting in a type II error. 


\section{Inappropriate shock}

Inappropriate therapy reached the significance level for the Social limitation domain and the overall summary score. While it was clear that this subgroup in our cohort scored generally worse (difference in mean overall summary score - 21.8), the number of patients in this subgroup was small $(n=5)$. Due to this, the magnitude of decrease in KCCQ-12 score is uncertain. However, several mechanisms that could lead to lower health status in these patients exist, such as anxiety after painful shocks or them serving as a marker of rapid atrial fibrillation, the most common reason for inappropriate shocks [15]. It has been shown that inappropriate shocks due to atrial fibrillation, but not due to lead failure, are associated with increased all-cause mortality [15].

\section{Atrial fibrillation}

In our cohort, a history of atrial fibrillation before ICD implant predicted significantly lower KCCQ-12 scores at follow-up (difference in mean overall summary score-10.9). There was a statistically significant reduction of similar size in both Symptom frequency and Social limitation, as well as a trend toward the same in other domains. Atrial fibrillation is associated with marked decreases in health-related quality of life [16]. In patients with heart failure and concomitant atrial fibrillation, therapy with catheter ablation improves healthrelated quality of life as well as heart failure specific health status measured by the MLHFQ [17]. The association between atrial fibrillation and lower health status as measured by the KCCQ-12 in heart failure patients could be due to atrial fibrillation being more common in severe heart failure, atrial fibrillation leading to a deterioration of left ventricular systolic function over time, or atrial fibrillation and heart failure both contributing to the burden of disease in ways that the KCCQ-12 cannot discriminate between [18].

\section{Complications requiring surgery}

There were no statistical differences in any domain or in the KCCQ-12 overall summary score. Lead-related problems are the most common complications that requires new surgery, after 10 years $25 \%$ of leads have been affected by complications [19]. In our whole ICD cohort, lead dislodgement or lead dysfunction constituted $60 \%$ of complications requiring surgery [7]. Therefore, while KCCQ-12 scores predict several outcomes, it is likely that lead-related complications depend more on lead-related factors rather than health status. In one study on patients with an ICD and hypertrophic cardiomyopathy, complications requiring surgery did not affect health-related quality of life measured by the SF-36 [20]. However, in that study as well as in the current study, follow-up time was long, it is plausible that complications requiring surgery would affect health status in the short term. This should be evaluated in future trials.

\section{Strengths and weaknesses}

This cross-sectional observational study provides realworld data regarding KCCQ-12 scores in an unselected cohort of patients with primary prevention ICD due to heart failure with long-term follow-up. All patients and variables have been validated from electronic medical records by physicians. The total number of patients was relatively low making interpretation more difficult. Few patients in some subgroups make some of the subgroup analyses prone to type I and II error. While the response rate of $71 \%$ was considered acceptable, $29 \%$ of patients did not return the questionnaire thus there is a risk of selection bias. Our study shares the limitation of a low proportion of females seen in numerous ICD cohorts. It is unknown to which extent geographical differences in the ICD management within a country matters and if interpretations can be generalized to other countries.

\section{Clinical perspective}

This observational pragmatic study demonstrates a patient-reported health status which is lower than in some prospective studies including randomized controlled trials. Still, this study indicates an acceptable health status in long-term survivors after ICD-implant. The selection of ICD candidates seems to be adequate as the health status likely can be translated into moderate burden of symptoms. Nevertheless, this study highlights the importance of optimal medical management. Therefore, modern optimal heart failure medication and structured clinical care is warranted in addition to device follow-up.

\section{Conclusions}

In a real-world pragmatic setting, primary prevention ICD patients with heart failure report an acceptable disease-specific health status according to KCCQ-12 at long-term follow-up. Ischemic and nonischemic underlying etiology showed similar health status whereas atrial fibrillation was associated with worse outcome.

\section{Abbreviations}

CRT-D: Cardiac resynchronization therapy defibrillator; ESC: European Society of Cardiology; ICD: Implantable cardioverter defibrillator; KCCQ: Kansas City Cardiomyopathy Questionnaire; KCCQ-12: 12-Item Kansas City Cardiomyopathy Questionnaire; MLHFQ: Minnesota Living with Heart Failure Questionnaire; NYHA class: New York Heart Association functional classification. 


\section{Acknowledgements}

Thanks to Kristina Bergström for her assistance with administration of the questionnaires. Region Gävleborg funded this research project.

\section{Authors' contributions}

GM: design, collection and interpretation of data, statistical analyses, writing of the article. MW: interpretation of data, critical revision. PM: design, collection and interpretation of data, statistical analyses, critical revision. All authors read and approved the final manuscript.

\section{Funding}

Open access funding provided by Uppsala University. Region Gävleborg funded this research project through GM and PMs employments but had no direct involvement in the design of the study or collection, analysis, and interpretation of data or the writing of the manuscript.

\section{Availability of data and materials}

Upon request.

\section{Declarations}

\section{Ethics approval and consent to participate}

The study complies with the Declaration of Helsinki and has been approved by the Ethical Review Board in Uppsala (document number 2018/416). Written informed consent was obtained from all individual participants included in the study.

\section{Consent for publication}

Informed consent was obtained from all individual participants included in the study.

\section{Competing interests}

GM has received speaker fees from Alnylam, Internetmedicin, and MSD. PM has received speaker fees or grants from Abbott, Alnylam, Amicus Therapeutics, Bayer, AstraZeneca, BMS, Boehringer-Ingelheim, Internetmedicin, Lilly, MSD, Novo Nordisk, Octopus Medical, Pfizer, Vifor Pharma, and Zoll. No external financial support or funding was received for this study. MW discloses no conflict of interest.

\section{Author details}

${ }^{1}$ Centre for Research and Development, Uppsala University/Region Gävleborg, 80187 Gävle, Sweden. ²Department of Building Engineering, Energy Systems and Sustainability Science, University of Gävle, 80176 Gävle, Sweden. ${ }^{3}$ Cardiology Research Unit, Department of Medicine, Karolinska Institutet, 171 76 Stockholm, Sweden.

Received: 17 February 2021 Accepted: 20 August 2021

Published online: 28 August 2021

\section{References}

1. Priori SG, Blomström-Lundqvist C, Mazzanti A, et al. 2015 ESC guidelines for the management of patients with ventricular arrhythmias and the prevention of sudden cardiac death: The Task Force for the Management of Patients with Ventricular Arrhythmias and the Prevention of Sudden Cardiac Death of the European Society of Cardiology (ESC). Endorsed by: Association for European Paediatric and Congenital Cardiology (AEPC). Eur Heart J. 2015;36:2793-867. https://doi.org/10.1093/eurheartj/ehv316.

2. Ponikowski P, Voors AA, Anker SD, et al. 2016 ESC guidelines for the diagnosis and treatment of acute and chronic heart failure: The Task Force for the diagnosis and treatment of acute and chronic heart failure of the European Society of Cardiology (ESC) developed with the special contribution of the Heart Failure Association (HFA) of the ESC. Eur Heart J. 2016;37:2129-200. https://doi.org/10.1093/eurheartj/ehw128.

3. Anker SD, Agewall S, Borggrefe M, et al. The importance of patientreported outcomes: a call for their comprehensive integration in cardiovascular clinical trials. Eur Heart J. 2014;35:2001-9. https://doi.org/ 10.1093/eurheartj/ehu205.

4. Green $C P$, Porter CB, Bresnahan DR, et al. Development and evaluation of the Kansas City Cardiomyopathy Questionnaire: a new health status measure for heart failure. J Am Coll Cardiol. 2000;35:1245-55. https://doi. org/10.1016/s0735-1097(00)00531-3.

5. Spertus JA, Jones PG. Development and validation of a short version of the Kansas City Cardiomyopathy Questionnaire. Circ Cardiovasc Qual Outcomes. 2015;8:469-76. https://doi.org/10.1161/CIRCOUTCOMES.115. 001958.

6. Yee D, Novak E, Platts A, et al. Comparison of the Kansas City Cardiomyopathy Questionnaire and Minnesota Living with Heart Failure Questionnaire in predicting heart failure outcomes. Am J Cardiol. 2019;123:807-12. https://doi.org/10.1016/j.amjcard.2018.11.037.

7. Mattsson G, Magnusson P. Long-term follow-up of implantable cardioverter defibrillator patients with regard to appropriate therapy, complications, and mortality. Pacing Clin Electrophysiol. 2020;43:245-53. https:// doi.org/10.1111/pace.13869.

8. Spertus J, Peterson E, Conard MW, et al. Monitoring clinical changes in patients with heart failure: a comparison of methods. Am Heart J. 2005;150:707-15. https://doi.org/10.1016/j.ahj.2004.12.010.

9. Veazie PJ, Noyes K, Li Q, et al. Cardiac resynchronization and quality of life in patients with minimally symptomatic heart failure. J Am Coll Cardiol. 2012;60:1940-4. https://doi.org/10.1016/j.jacc.2012.06.054.

10. Bundgaard JS, Thune JJ, Nielsen JC, et al. The impact of implantable cardioverter-defibrillator implantation on health-related quality of life in the DANISH trial. Europace. 2019;21:900-8. https://doi.org/10.1093/europ ace/euz018.

11. Khariton $Y$, Nassif ME, Thomas $L$, et al. Health status disparities by sex, race/ ethnicity, and socioeconomic status in outpatients with heart failure. JACC Heart Fail. 2018;6:465-73. https://doi.org/10.1016/j.jchf.2018.02.002.

12. Sticherling C, Arendacka B, Svendsen JH, et al. Sex differences in outcomes of primary prevention implantable cardioverter-defibrillator therapy: combined registry data from eleven European countries. Europace Eur Pacing Arrhythmias Cardiac Electrophysiol J Work Groups Cardiac Pacing Arrhythmias Cardiac Cell Electrophysiol Eur Soc Cardiol. 2018;20:963-70. https://doi.org/10.1093/europace/eux176.

13. Garay A, Tapia J, Anguita M, et al. Gender differences in health-related quality of life in patients with systolic heart failure: results of the VIDA multicenter study. J Clin Med. 2020;9:2825. https://doi.org/10.3390/jcm9092825.

14. Sears SF, Rosman L, Sasaki S, et al. Defibrillator shocks and their effect on objective and subjective patient outcomes: results of the PainFree SST clinical trial. Heart Rhythm. 2018;15:734-40. https://doi.org/10.1016/j. hrthm.2017.12.026.

15. Kleemann T, Hochadel M, Strauss M, et al. Comparison between atrial fibrillation-triggered implantable cardioverter-defibrillator (ICD) shocks and inappropriate shocks caused by lead failure: different impact on prognosis in clinical practice. J Cardiovasc Electrophysiol. 2012;23:735-40. https://doi.org/10.1111/j.1540-8167.2011.02279.x.

16. Thrall G, Lane D, Carroll D, et al. Quality of life in patients with atrial fibrillation: a systematic review. Am J Med. 2006;119:448.e1-19. https://doi.org/ 10.1016/j.amjmed.2005.10.057.

17. Briceño DF, Markman TM, Lupercio F, et al. Catheter ablation versus conventional treatment of atrial fibrillation in patients with heart failure with reduced ejection fraction: a systematic review and meta-analysis of randomized controlled trials. J Interv Cardiac Electrophysiol Int J Arrhythmias Pacing. 2018:53:19-29. https://doi.org/10.1007/s10840-018-0425-0.

18. Steinberg BA, Piccini JP. Tackling patient-reported outcomes in atrial fibrillation and heart failure: identifying disease-specific symptoms? Cardiol Clin. 2019;37:139-46. https://doi.org/10.1016/j.ccl.2019.01.013.

19. Koneru JN, Jones PW, Hammill EF, et al. Risk factors and temporal trends of complications associated with transvenous implantable cardiac defibrillator leads. J Am Heart Assoc. 2018;7:e007691. https://doi.org/10. 1161/JAHA.117.007691

20. Magnusson P, Mörner S, Gadler F, et al. Health-related quality of life in hypertrophic cardiomyopathy patients with implantable defibrillators. Health Qual Life Outcomes. 2016;14:62. https://doi.org/10.1186/ s12955-016-0467-x.

\section{Publisher's Note}

Springer Nature remains neutral with regard to jurisdictional claims in published maps and institutional affiliations. 